\title{
Adolescentes en riesgo: Búsqueda de sensaciones, adicción al internet y procrastinación ${ }^{3}$
}

\section{Marisol Morales Rodríguez}

PhD. en Ciencias de la Educación Universidad Michoacana de San Nicolás de Hidalgo Morelia Michoacán, México

Correo electrónico: marisolmoralesrodriguez@gmail.com
Recibido: 29/03/2020

Evaluado: $21 / 05 / 2020$

Aceptado: 03/06/2020

\section{Resumen}

El objetivo del estudio fue caracterizar las conductas de riesgo búsqueda de sensaciones, adicción al internet y procrastinación en adolescentes y determinar la relación entre las variables de estudio. El método se enmarca en el enfoque cuantitativo, diseño no experimental, alcance descriptivo-correlacional. Participaron 702 adolescentes escolarizados con edad promedio de 14.9 años. Los instrumentos utilizados fueron la Escala Búsqueda de sensaciones, Forma $V$ (Zuckerman, Eysenck \& Eysenck, 1978; adaptación de Pérez \& Torrubia, 1986), Cuestionario de Experiencias Relacionadas con el Internet de Beranuy, Chamarro, Graner y Carbonell (2009) y Escala de Procrastinación General (Busko, 1998), aplicándose previo consentimiento de los padres. Los resultados destacan que los adolescentes incurren en conductas de riesgo como búsqueda de experiencias novedosas y arriesgadas, adicción al internet y presentan conductas dilatorias. Tales conductas se encuentran vinculadas entre sí, colocando al adolescente en una condición altamente vulnerable, que puede generar consecuencias nocivas en su salud psicológica.

\section{Palabras clave}

Conductas de riesgo, Búsqueda de sensaciones, Adicción al internet, Procrastinación, Adolescentes.

3 Para citar este artículo: Morales-Rodríguez, M. (2022). Adolescentes en riesgo: Búsqueda de sensaciones, adicción al internet y procrastinación. Informes Psicológicos, 22(1), pp. 43-60 http://dx.doi.org/10.18566/infpsic.v22n1a03 


\title{
Teens at Risk: Sensation-Seeking, Internet Addiction, and Procrastination
}

\begin{abstract}
The objective of the study was to characterize risky behaviors such as sensation-seeking, internet addiction and procrastination in adolescents and to determine the relationship between the study variables. The method is framed in the quantitative approach, non-experimental design, and descriptivecorrelational scope. 702 adolescents enrolled in school with an average age of 14.9 years participated. The instruments used were the Sensation Search Scale, Form V (Zuckerman, Eysenck \& Eysenck, 1978; adaptation of Pérez \& Torrubia, 1986), Questionnaire of Experiences Related to the Internet by Beranuy, Chamarro, Graner and Carbonell (2009), and General Procrastination Scale (Busko, 1998), applied with the prior consent of the parents. The results highlight that adolescents engage in risky behaviors such as searching for new and risky experiences, internet addiction; as a result, they present delaying behaviors. Such behaviors are linked to each other, placing the adolescent in a highly vulnerable condition, which can generate harmful consequences on their psychological health.
\end{abstract}

Keywords

Risky behaviors, Sensation-seeking, Internet addiction, Procrastination, Adolescents.

\section{Adolescentes em risco: busca de sensações, vício em Internet e procrastinação}

Resumo

0 objetivo do estudo foi caracterizar os comportamentos de risco relacionados à busca de sensações, vício em internet e procrastinação em adolescentes, determinando a relação entre as variáveis do estudo. 0 método faz parte daabordagem quantitativa, com desenho não experimental e escopo descritivo-correlacional. Participaram 702 adolescentes matriculados na escola com idade média de 14,9 anos. 0s instrumentos utilizados foram Sensation Search Scale, Form V (Zuckerman, Eysenck \& Eysenck, 1978; adaptação de Pérez \& Torrubia, 1986), Questionário de Experiências Relacionadas à Internet de Beranuy, Chamarro, Graner e Carbonell (2009) e Escala General Procrastinação (Busko, 1998), aplicada com o consentimento prévio dos pais. Os resultados destacam que os adolescentes se envolvem em comportamentos de risco, como busca de novas experiências de risco, dependência de internet, apresentando comportamentos dilatórios. Tais comportamentos estão ligados entre si, colocando o adolescente em uma condição de alta vulnerabilidade, o que pode gerar consequências prejudiciais à sua saúde psicológica.

Palavras chave

Comportamentos de risco, Busca de sensações, Dependência em Internet, Procrastinação, Adolescentes. 


\section{ntroducción}

La adolescencia se concibe como una fase del ciclo vital caracterizada por una serie de transformaciones que son el precedente de la asunción de un rol adulto, dichas transformaciones se viven como duelos y pérdidas, lo que provoca una crisis; sin embargo, una crisis esperada durante la adolescencia puede convertirse en riesgo si se conjugan una serie de elementos personales, familiares y sociales que vulneren la integridad del adolescente.

Barcelata (2015) afirma que el riesgo es un conjunto de variables personales y del medio que preceden a un comportamiento desadaptado; en este sentido, se consideran como indicadores de riesgo el nivel socioeconómico, sucesos estresantes, situaciones adversas como enfermedades, separaciones, violencia, incluso desastres naturales, entre otros.

No obstante, también existen factores personales que constituyen un riesgo para el adolescente como la inmadurez cerebral, las deficiencias en el autocontrol y en la resolución de problemas, el egocentrismo y la incesante búsqueda del placer que lo lleva a conductas extremas.

Los cambios en todas las áreas del desarrollo hacen al adolescente mucho más susceptible a la influencia de los pares, a la reorganización familiar y los fenómenos sociales y económicos, de tal forma que aumenta el riesgo de desarrollar patrones de comportamiento desadaptativos.

Las conductas de riesgo son aquellas que tienen consecuencias perjudiciales para la persona que las lleva a cabo y para quienes le rodean; dichas repercusiones merman tanto la salud física como psicológica (Orcasita, Mosquera \& CarriIlo, 2018).

Por lo regular, dichas conductas en la etapa adolescente son un reflejo de la falta de madurez frente a los abrumadores cambios, la necesidad de ser independiente y la lucha frente a la autoridad, la sensación de invencibilidad, entre otras, lo que constituye un peligro para la integridad del mismo adolescente y de quienes lo rodean.

En esta línea, diversos estudios como el de Rosabal, Romero, Gaquin y Hernández (2015) afirman que las conductas de riesgo son suscitadas por diversos condicionantes, entre los que sobresalen la urgencia del adolescente por buscar experiencias excitantes y arriesgadas, aunado a las deficiencias en el autocontrol que llevan a transgredir normas familiares y sociales, y a postergar actividades previamente planificadas. No obstante, no se puede dejar de lado la participación de factores de índole social, como la presión de iguales, un ambiente familiar disfuncional y un entorno sociocultural desfavorecido, entre otros.

La desestimación del riesgo en la adolescencia tiene un origen multifactorial, resultado de los cambios cognitivos, emocionales y sociales, a los que se suma lo que Oliva (2007) ha señalado como inmadurez cerebral, es decir, el comportamiento adolescente obedece a una limitada participación de la corteza prefrontal, estructura en proceso de desarrollo, lo que se traduce en la búsqueda de recompensas inmediatas y de experiencias placenteras. 


\section{Búsqueda de sensaciones}

La búsqueda de sensaciones se conceptualiza como la necesidad que muestra el adolescente por involucrarse en experiencias poco usuales e intensas, a la vez de asumir riesgos innecesarios a fin de experimentar placer (Zuckerman, como se citó en Palacios, 2015).

Dicho patrón suele convertirse en una conducta de riesgo por la incapacidad del adolescente de controlarse, de implicarse en riesgos innecesarios por el simple deseo del disfrute, sin pensar en las consecuencias de sus acciones, de tal forma que este patrón puede dar origen a otras problemáticas mayores como el uso ilícito de sustancias, sexo precoz, trastornos alimentarios, conductas violentas, delincuencia, entre otras.

La búsqueda de sensaciones se concibe como rasgo de personalidad ya que, independientemente de la etapa del ciclo vital, algunas personas son más proclives a presentar dicha condición, como resultado de una necesidad manifiesta de explorar y vivir experiencias intensas, fuera de la rutina, lo cual suele ser más común durante la adolescencia.

Dicho constructo ha sido retomado en diversos estudios que abordan la propensión a tomar riesgos en una amplia gama de comportamientos, los cuales llevan a la asunción de conductas que ponen en peligro al adolescente. Con base en ello, se le asocia no solo con la necesidad de experiencias excitantes, sino que se incluye un componente de riesgo persistente (Latorre, Cámara \& García, 2014).
De acuerdo con las predicciones de algunos modelos de conductas de salud, las personas que perciben más riesgos en llevar a cabo una conducta dada, tienden a apartarse mientras que las personas que perciben pocos riesgos pueden implicarse sin problema alguno. No obstante, resultados de diversas investigaciones han arrojado conclusiones contradictorias sobre la relación entre percepción de riesgo y conducta arriesgada. Lo anterior podría sustentarse en que, si no se experimentan consecuencias negativas después de una conducta de riesgo, pueden cambiar las creencias de las personas sobre el peligro real de dicha conducta. Y, en el caso particular de los adolescentes, si pocas veces se enfrentan a consecuencias desagradables en torno a la conducta problema, su estimación de riesgo es baja (González-lglesias, Gómez-Fraguela, Gras \& Planes, 2014).

Los mismos autores afirman que la relación entre la búsqueda de sensaciones y la conducta arriesgada se basa en la percepción del riesgo. Si las personas perciben menos riesgos, tienden a puntuar alto en búsqueda de sensaciones, involucrándose en mayores conductas problema, destacando una disposición personal a asumir riesgos.

Una hipótesis alternativa, en palabras de Horvath y Zuckerman (1996), este último teórico, pionero en el estudio de la búsqueda de sensaciones, es que las personas con dicho rasgo presentan lo que se ha denominado "sesgo optimista", percibiendo menos posibilidades de resultados negativos ante la conducta de riesgo. 
El sesgo optimista es un reflejo de la fábula personal, caracterizado por la creencia errónea del adolescente de ser invencible y de que no será víctima de las circunstancias, actuando sin evaluar las consecuencias de sus actos en la búsqueda de nuevas experiencias y sensaciones, por lo que es más probable que se involucre en conductas de riesgo.

Es importante aclarar que aunque la conceptualización de la búsqueda de sensaciones destaca la voluntad de asumir riesgos, esto se da solamente por las recompensas que estimulan a la persona, como la novedad o la intensidad, pero no se compara en absoluto con la búsqueda de riesgos (Boyle, Saklofske, \& Matthews, 2015).

Palacios (2015) tuvo a bien diferenciar el constructo en cuestión, destacando las posturas de los principales teóricos que lo han abordado. Para Arnett (1994), la búsqueda de sensaciones se basa en las dimensiones de novedad e intensidad, y enfatiza que la socialización es la base de dicho rasgo. Zuckerman, en 2007 destacó además de la necesidad de experimentar lo emergente y novedoso, la asunción de riesgos, y rescató la existencia de bases biológicas en dicho patrón de comportamiento. En tanto, Roth, Hammelstein y Brähler (2007) afirmaron que la búsqueda de sensaciones es una necesidad de estimulación.

Como se observa, la búsqueda de sensaciones es un patrón que se conforma de conductas específicas como impulsividad, desinhibición, deseo de buscar experiencias nuevas e intensas, evitar la monotonía y enfrentarse al riesgo. En dicho patrón confluyen bases biológicas asociadas al temperamento, y bases sociales que refuerzan su ocurrencia y moldean la manera como el adolescente responde. La relevancia de su estudio radica en que se asocia con la presencia de otras conductas de riesgo como una reacción en cadena, esto es, la necesidad de vivir experiencias intensas lleva al adolescente a asumir mayores riesgos.

En este orden de ideas, la necesidad de experimentar sensaciones se asocia con la falla en el control de impulsos, y, en palabras de Silva-Ortega y ZambranoVillalba (2018), uno de los componentes fundamentales de los trastornos adictivos es precisamente la pérdida de control, de ahí que lo relevante en las adicciones es la relación negativa y destructiva que se establece con la actividad que genera la dependencia, que lleva a la persona a mostrarse incapaz de controlar.

Cabe destacar que las conductas adictivas ya no solo se asocian con el abuso de sustancias químicas, sino que también se manifiestan en aspectos psicológicos.

\section{Adicciones psicológicas: Adicción al internet}

El adolescente es vulnerable a las adicciones, debido a las dificultades en el autocontrol, como resultado de los cambios que experimenta en esta etapa del desarrollo. Dichas transformaciones pueden generar una sensación de vacío que lo lleve a la búsqueda de algo que lo "llene", y muchas veces, es la necesidad de afecto, de atención de los padres, de certidumbre, optando por llenarlo con objetos y con conductas específicas de riesgo. 
Las adicciones psicológicas hacen referencia a un conjunto de comportamientos que se repiten sin que las personas puedan hacer algo por controlarlos. Este hecho les genera sensaciones de incapacidad para manejar la situación en la que se encuentran, ya que requieren dedicarle más tiempo a estas actividades para librarse de las sensaciones desagradables que le produce el hecho de no realizarlas (Salas, 2014, pp. 112-113).

Entre las adicciones psicológicas destacan aquellas referidas a las nuevas tecnologías, las cuales son más comunes en adolescentes y jóvenes. Desafortunadamente, este tipo de adicción ha crecido exponencialmente en los últimos años, reemplazando a otro tipo de conductas problema, debido a que en parte se han minimizado sus consecuencias nocivas, ya que se piensa erróneamente que, por el hecho de no ingerir o consumir alguna sustancia, la adicción a las tecnologías no representa una dependencia, sino más bien se identifica como una conducta que, aunque se sabe incómoda, es parte de los patrones de comportamiento más comunes.

La tecnología ha facilitado nuestras vidas y ha permitido contar con información veraz, oportuna y rápida, eso es lo que la hace atractiva: la recompensa inmediata; no obstante, a pesar de mantenernos conectados con todos y todo, al mismo tiempo no se contacta con alguien en particular.

Actualmente, se han utilizado diversas denominaciones para definir la adicción al internet como "trastorno adictivo a la internet", "uso problemático de internet", "uso patológico de internet" y "uso excesivo de Internet", sin embargo, lo que define dicha condición es el uso incontrolable de la internet, que lleva a que la persona descuide otras áreas importantes de su vida (Silva-Ortega \& Zambrano-Villalba, 2018).

El problema de esta adicción radica en que la tecnología se ha apropiado de la vida de las personas, particularmente los jóvenes, emergiendo como la vía rectora de acercamiento con las principales redes de apoyo social, pero no para favorecer la comunicación, sino simplemente para conectar con otros (Castaño \& Páez, 2019).

Parra et al. (2016) señalan que la incapacidad de la persona de controlar el uso del internet lleva a la experimentación de síntomas ansiosos e irritabilidad cuando no accede a las redes, y la situación se agudiza al observarse el descuido de rutinas diarias, la afectación del sueño nocturno, dejar de comer y mostrar desinterés por otros temas, todo por permanecer conectados. En este sentido, la adicción al internet, como lo señalan Rojas-Jara, Ramos-Vera, Pardo-González y HenríquezCaroca (2018) puede llevar a un deterioro emocional y psicológico de la persona.

Resulta preocupante que el adolescente se aísle y se sumerja en una realidad virtual, ya que deja de lado, no solo actividades escolares o aquellas propias de casa, sino también la convivencia familiar, las relaciones cara a cara con sus coetáneos o con adultos fuera del núcleo familiar, es decir, se deteriora su capacidad para interactuar con otros. Dichas acciones afectan el desarrollo psicosocial del adolescente, ya que un indicador importante de ajuste psicológico es precisamente el desarrollo de habilidades sociales. 
Al respecto, Silva-Ortega y ZambranoVillalba (2018) afirman que la adicción a internet representa una compensación ante un déficit en la personalidad del adicto, y, dado que es un medio de comunicación, se convierte en un sustituto de la vida real en personas introvertidas o con deficiencias en las habilidades sociales.

En la misma línea, Gámez y Villa (2015) enfatizan una serie de características en aquellas personas que han creado una dependencia hacia el internet como escasas habilidades sociales, lo que lleva a una mayor tendencia al aislamiento, temperamento más impulsivo, conectarse para aliviar sentimientos de tristeza, ansiedad y soledad. Lo anterior tendría relación con la necesidad de buscar sensaciones novedosas como mecanismo compensatorio.

La dependencia al Internet, además de ir acompañada de otras problemáticas de naturaleza similar, deteriora la vida de las personas, ya que se ven afectadas diversas actividades apareciendo también conductas dilatorias.

Mantenerse en el mundo virtual resulta altamente placentero y estimulante, no obstante, cuando se navega se pierde la noción del tiempo. Ramos-Galarza, Jadan-Guerrero, Paredes-Núñez, BolañosPasqueld y Gómez-García (2017) aseveran que el internet es el principal medio tecnológico en donde los jóvenes consumen sin mayor beneficio la mayor parte del tiempo. Pasan de plataformas virtuales de aprendizaje a las redes sociales, páginas de entretenimiento y reproductores de películas, principalmente, lo que se convierte en estímulos que aumentan el comportamiento procrastinador.

\section{Procrastinación}

La procrastinación hace referencia a una tendencia de aplazar el inicio y/o finalización de tareas que se han planificado previamente para ser realizadas en un tiempo dado. Se acompaña de malestar subjetivo debido no solo al manejo del tiempo sino a la dificultades en la autoregulación (Díaz-Morales, 2019).

Dicho patrón conductual merma la iniciativa y la proactividad, ya que no se logra concretar lo previamente planeado, incluso puede llevar a una conducta deficiente en cuanto a estrategias efectivas de afrontamiento.

El malestar ocasionado por la acción de aplazar lleva a experimentarla internamente como una carga de emociones negativas y de tensión. Pero, en un sentido inverso, existe un componente emotivo en la conducta procrastinadora, ya que la tarea a realizar puede representar un afecto displacentero y resultar altamente estresante, aumentando la probabilidad de emociones negativas que fomenten la inmovilidad (Moreta-Herrera, DuránRodríguez \& Villegas-Villacrés, 2018).

La acción de postergar pudiera ser más común en la adolescencia debido a la necesidad de adaptarse a los cambios, al bajo control de impulsos, a la necesidad de buscar experiencias estimulantes, a las diferencias entre el tiempo conceptual y vivencial y, en general, a una baja capacidad de autorregulación.

El patrón de la conducta dilatoria genera severas consecuencias durante la adolescencia, repercutiendo negativamente en el aspecto académico ya que los adolescentes postergan sus tareas 
escolares por otras actividades de mayor interés como los juegos, las fiestas, reunirse con los pares, las redes sociales, entre otros; si en esta etapa, estos hábitos quedan instaurados, no solamente afectará el área académica, sino que, a la larga, se verá reflejado en diversas actividades (Atayala \& García, 2019).

Los esfuerzos de la comunidad científica por estudiar la procrastinación se encaminan no solo en conocer los factores de riesgo asociados, sino en proponer estrategias de intervención que, si no la erradican, al menos disminuya su incidencia, ya que se ha convertido en un problema recurrente, no solo en adolescentes sino en todos los sectores de la población, trayendo consigo afectaciones en la familia, con los compañeros y amigos, en el trabajo y, en general, en las relaciones que se establecen en los diferentes contextos de desarrollo. El hecho de que se haya instalado como un comportamiento común no lo exime de que sea poco adaptativo, al contrario, deja al descubierto que los padres, educadores, los diferentes profesionales y la sociedad en su conjunto han sido rebasados por dicho fenómeno.

Los planteamientos de Atayala y García (2019) resaltan que la procrastinación implica un aplazamiento de los compromisos personales, a pesar de tener presentes las consecuencias negativas; ello coloca al adolescente en desventaja frente al proceso del logro de identidad, al favorecer una baja autoeficacia, un pobre autocontrol y un estado de insatisfacción en general, dejando pasar las oportunidades e inmovilizándose.

Los constructos anteriormente descritos tienen el común denominador de que son problemáticas actuales y que van en aumento; es así como surge el objetivo del presente estudio, el cual pretende caracterizar las conductas de riesgo búsqueda sensaciones, adicción al internet y procrastinación en adolescentes y determinar si existe relación entre éstas.

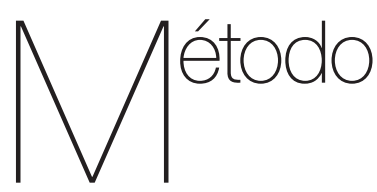

La investigación se basa en una metodología cuantitativa, bajo un diseño no experimental, transversal, cuyo alcance es descriptivo-correlacional.

\section{Participantes}

La muestra de estudio se constituyó por 702 adolescentes de nivel básico, en particular secundaria y nivel medio superior de distintas escuelas públicas de Morelia, Michoacán, México. El rango de edad de los participantes oscila entre los 13 y 18 años ( $M=14.9$; $D E=1.7)$. $E l$ $46.8 \%$ de la muestra pertenece al nivel secundaria y el $53.2 \%$ a preparatoria, con una Desviación estándar de 1.42. En relación al género, la distribución fue de un $51.3 \%$ de mujeres y el $48.7 \%$ de hombres $(\mathrm{DE}=.60)$.

Los criterios de inclusión fueron que los adolescentes pertenecieran a las instituciones educativas seleccionadas y que su participación fuera voluntaria; así mismo, se excluyó a aquellos que se negaron a participar y se descartó a los que no completaron los instrumentos de evaluación. 


\section{Instrumentos}

Los instrumentos empleados fueron tres. La Escala Búsqueda de Sensaciones (Forma V) (Zuckerman, 1978; adaptación de Pérez y Torrubia, 1986), consta de 40 ítems, con opciones de respuesta dicotómicas, distribuidos en cuatro subescalas: a) Búsqueda de emociones: "algunas veces me gusta hacer cosas que impliquen pequeños sobresaltos"; b) Búsqueda de excitación: "he probado marihuana $u$ otras hierbas o me gustaría hacerlo"; c) Desinhibición: "me gusta tener experiencias y sensacio nes nuevas y excitantes aunque sean poco convencionales o incluso ilegales"; y d) Susceptibilidad al aburrimiento: "no tengo paciencia con las personas grises o aburridas". El índice de consistencia interna de la escala total oscila en un rango de .83 a .86 .

El Cuestionario de Experiencias Relacionadas con el Internet (CERl; Beranuy, Chamarro, Graner \& Carbonell, 2009) conformado por 10 ítems que se valoran mediante una escala tipo Likert con cuatro opciones ( 1 = nada, hasta $4=$ bastante), los cuales están categorizados en dos factores: Intrapersonales que incluye reactivos como "¿Te enfadas o te irritas cuando alguien te molesta mientras estás conectado?”, “Cuando no estás conectado a Internet te sientes nervioso o preocupado?"; y Factores Interpersonales, con reactivos como los siguientes: "¿Con qué frecuencia abandonas las cosas que estás haciendo para estar más tiempo conectado a la red?", “Con qué frecuencia haces nuevos amigos en línea?". El primer factor cuenta con una consistencia interna de .74 y el segundo de .75. El índice de confiabilidad total es de .77 .
La Escala de Procrastinación General de Busko (1998) es unifactorial y está conformada por 13 ítems con opciones de respuesta tipo Likert de cinco puntos (1= siempre; 2=casi siempre; $3=a$ veces; $4=$ pocas veces; 5=nunca). Algunos ejemplos de los reactivos son: "tiendo a perder mucho el tiempo"; "aplazo la toma de decisiones difíciles". El índice de consistencia interna es de .82.

\section{Procedimiento}

Para llevar a cabo la investigación, se consideraron instituciones públicas de nivel básico y nivel medio superior de diferentes sectores de la ciudad de Morelia, Michoacán, México; sin embargo, el muestreo no fue probabilístico debido a que solo algunas de ellas consintieron participar en la investigación. Cabe aclarar que participaron escuelas de los 4 sectores primarios en los que se divide la ciudad. En cada una de las instituciones de nivel secundaria y preparatoria, las autoridades educativas determinaron los grupos a los cuales se aplicarían los instrumentos, por lo que se optó por un muestreo no probabilístico intencional. La aplicación de los instrumentos se realizó de manera colectiva dentro de las aulas a cargo de alumnos de la Facultad de Psicología de la Universidad Michoacana de San Nicolás de Hidalgo que colaboraban en la investigación, utilizando la versión impresa de las escalas; la aplicación tuvo una duración aproximada de 40 minutos.

Como parte de las consideraciones éticas, la investigación se apegó a los estándares establecidos para proyectos de investigación, haciendo uso del consentimiento informado, el cual fue enviado a los padres de familia a través de los 
estudiantes para que autorizaran su participación; así mismo, se hizo hincapié en el carácter confidencial de la información aportada y el respeto al anonimato de los participantes.

Para el análisis de los datos, una vez concentrada la información, se hizo uso del programa SPSS 22.0, realizando un análisis de frecuencias para la obtención de las medidas de tendencia central, prueba t de Student y Coeficiente de correlación de Pearson.

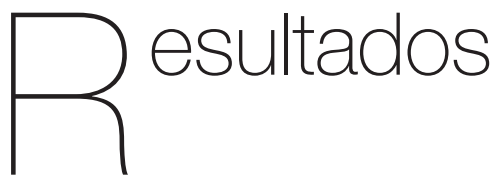

Los resultados que a continuación se presentan siguen la misma organización: búsqueda de sensaciones, adicción al internet y procrastinación; posteriormente, se describen las correlaciones entre las variables de estudio.

\section{a. Búsqueda de sensaciones}

Tabla 1.

Niveles de Búsqueda de Sensaciones

\begin{tabular}{lccc}
\hline & $\begin{array}{c}\text { Búsqueda de } \\
\text { Emociones }\end{array}$ & Desinhibición & Susceptibilidad \\
\cline { 2 - 4 } Alto & 46.3 & 33.7 & 39.9 \\
Promedio & 34.7 & 43.8 & 37.0 \\
Bajo & 19.0 & 22.5 & 23.1 \\
\hline
\end{tabular}

Nota: Los niveles se presentan en porcentajes; Fuente: Elaboración propia

En torno a esta variable, se observa que los niveles se encuentran predominantemente en el nivel alto a excepción del factor desinhibición, donde el mayor porcentaje se concentra en el nivel medio; los datos reflejan una condición de vulnerabilidad en los adolescentes al mostrar la necesidad de buscar emociones, aventuras y experiencias y ser ligeramente más susceptible al aburrimiento, como se observa en la Tabla 1.

En otra línea, se encontraron diferencias en función del sexo y nivel educativo. Por sexo se encontró que los hombres son quienes se involucran mayormente en este tipo de conductas de riesgo, particularmente en búsqueda de emociones y desinhibición (Ver Tabla 2).

Tabla 2.

Diferencias en Búsqueda de sensaciones por sexo

\begin{tabular}{lccccccc}
\hline & \multicolumn{3}{c}{ Mujeres } & \multicolumn{2}{c}{ Hombres } & & \\
\cline { 1 - 5 } Emoción & M & DS & M & DS & t & p \\
\cline { 2 - 5 } Excitación & 14.5 & 2.52 & 15.8 & 2.45 & 2.2 & .025 \\
Desinhibición & 14.8 & 1.9 & 14.9 & 1.89 & .59 & .350 \\
Susceptibilidad & 15.9 & 2.36 & 16.1 & 2.47 & 2.8 & .001 \\
\hline
\end{tabular}

Nota: Emoción: Búsqueda de emociones; M: Media, DS: Desviación Estándar. Significancia: $\mathrm{p} \leq .01$; Fuente: Elaboración propia

De acuerdo al nivel educativo de los participantes, los resultados arrojan diferencias en tres de los cuatro factores, como se observa en la Tabla 3, siendo los adolescentes de secundaria quienes se involucran mayormente en este patrón de comportamiento.

Tabla 3.

Diferencias en Búsqueda de sensaciones por nivel educativo

\begin{tabular}{lccccccc}
\hline \multicolumn{7}{c}{ Secundaria } & \multicolumn{3}{c}{ Preparatoria } \\
\cline { 2 - 7 } & M & DS & M & DS & t & p \\
\cline { 2 - 7 } Emoción & 15.3 & 2.4 & 13.5 & 2.6 & 3.08 & .004 \\
Excitación & 15.0 & 2.3 & 14.1 & 2.2 & 2.90 & .033 \\
Desinhibición & 17.1 & 2.1 & 15.9 & 2 & 2.75 & .050 \\
Susceptibilidad & 16.5 & 1.5 & 16.4 & 1.4 & .22 & .760 \\
\hline Nota: Emoción: Búsqueda de emociones; M: Media, DS: Desvia-
\end{tabular}

ción Estándar. Significancia: $p \leq .01$; Fuente: Elaboración propia 
b. Adicción al internet

Los resultados señalan que los adolescentes se ubican en una zona de riesgo al predominar un nivel alto en el factor intrapersonales y ligeramente por encima del promedio en el factor interpersonales (ver Tabla 4).

Tabla 4.

Niveles de Adicción al internet

\begin{tabular}{lcc}
\hline & Interpersonales & Intrapersonales \\
\hline Alto & 35.2 & 41.3 \\
Promedio & 34.5 & 31.6 \\
Bajo & 30.3 & 27.1 \\
\hline
\end{tabular}

Nota: Los resultados por niveles se presentan en porcentajes; Fuente: Elaboración propia

Así mismo, se reportan diferencias por sexo, destacando que las mujeres muestran mayor dependencia hacia la Red en el factor interpersonales con respecto a los hombres. Por nivel educativo no se encontraron diferencias (ver Tabla 5).

Tabla 5.

Diferencias en Adicción al internet por sexo

\begin{tabular}{ccccccc}
\hline & \multicolumn{7}{c}{ Mujeres } & \multicolumn{3}{c}{ Hombres } \\
& $\mathbf{M}$ & DS & $\mathbf{M}$ & DS & $\mathbf{t}$ & $\mathbf{p}$ \\
\cline { 2 - 7 } Interpersonales & 9.3 & 2.4 & 8.2 & 2.2 & 1.95 & .05 \\
Intrapersonales & 12.5 & 3.1 & 12.7 & 3.2 & 1.05 & .15 \\
\hline
\end{tabular}

Nota: Interpersonales= Conflictos interpersonales; Intrapersonales: Conflictos intrapersonales; M: M dia, DS: Desviación Estándar.

Significancia: $p \leq .01$; Fuente: Elaboración propia

\section{c. Procrastinación}

Con respecto a la conducta procrastinante, como se muestra en la Tabla 6 , los participantes refieren conductas perentorias ubicándose por encima del promedio, lo cual es preocupante dado el impacto, no solo a nivel académico, sino en el resto de las áreas de desarrollo.

Tabla 6.

Niveles de Procrastinación general

\begin{tabular}{lc}
\hline & Procrastinación \\
\hline Alto & 43.1 \\
Promedio & 38.5 \\
Bajo & 18.4 \\
\hline
\end{tabular}

Nota: Los resultados por nivel se presentan en porcentajes; Fuente: Elaboración propia

Otro dato relevante son las diferencias halladas por nivel educativo. Como se evidencia en la Tabla 7, los adolescentes de secundaria presentan mayores conductas de dilación, en comparación con los adolescentes de nivel medio superior.

Tabla 7.

Diferencias en Procrastinación por nivel educativo

\begin{tabular}{|c|c|c|c|c|c|c|}
\hline & Secun & laria & Prepa & atoria & & \\
\hline \multirow{2}{*}{ Procrastinación } & $M$ & DS & M & DS & $t$ & $p$ \\
\hline & 42.09 & 7.8 & 39.9 & 8.1 & 2.1 & .051 \\
\hline
\end{tabular}

Nota: M: Media, DS: Desviación Estándar. Significancia: $\mathrm{p} \leq .01$ Fuente: Elaboración propia

En cuanto a las correlaciones entre las variables de estudio, existe relación entre búsqueda de sensaciones, particularmente excitación, desinhibición y susceptibilidad, con el factor interpersonales de adicción al internet; esto es, conforme el adolescente se involucra en experiencias extremas, desarrolla conductas adictivas (ver Tabla 8).

Tabla 8.

Correlación entre Búsqueda de sensaciones y Adicción al internet

\begin{tabular}{lcccc}
\hline & Emociones & Excitación & Desinhibición & Susceptibilidad \\
\cline { 2 - 4 } Interpersonales & .119 & $.206^{* *}$ & $.220^{* *}$ & $.210^{\star *}$ \\
Intrapersonales & .110 & .095 & .169 & $.190^{\star *}$ \\
\hline
\end{tabular}

Nota: Interpersonales= Conflictos interpersonales; Intrapersonales: Conflictos intrapersonales Emoción: Búsqueda de emociones; Significancia: ${ }^{\star \star} p<$. 01; Fuente: Elaboración propia. 
De igual forma, se encontró asociación entre Búsqueda de sensaciones, de manera específica excitación, desinhibición y susceptibilidad con procrastinación, lo que indica que a mayor involucramiento del adolescente en experiencias extremas, mayor presencia de conductas procrastinantes (Ver Tabla 9).

Tabla 9.

Correlación entre Búsqueda de sensaciones y Procrastinación

\begin{tabular}{ccccc}
\hline & Emociones & Excitación & Desinhibición & Susceptibilidad \\
\cline { 2 - 4 } Procrastinación & $.171^{* *}$ & $.210^{* *}$ & $.269^{* *}$ & $.247^{* *}$ \\
\hline
\end{tabular}

Nota: Emoción: Búsqueda de emociones; Significancia: ${ }^{\star \star} p<.01$; Fuente: Elaboración propia

En la misma línea, se registran correlaciones entre la adicción al internet en los factores interpersonales e intrapersonales y la procrastinación, lo que significa que cuanto mayor sea la adicción al internet, los adolescentes presentarán mayores conductas de postergación.

Tabla 10.

Correlación entre Adicción al internet y Procrastinación.

\begin{tabular}{lcc}
\hline & \multicolumn{1}{c}{ Interpersonales } & Intrapersonales \\
\cline { 2 - 3 } Procrastinación & $.472^{\star *}$ & $.375^{* *}$ \\
\hline
\end{tabular}

Nota: Interpersonales = Conflictos interpersonales; Intrapersonales: Conflictos intrapersonales; Significancia: ${ }^{\star \star} p<.01$;

Fuente: Elaboración propia

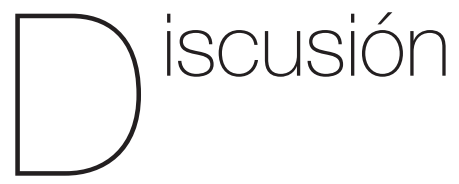

El objetivo de la presente investigación fue caracterizar las conductas de riesgo búsqueda sensaciones, adicción al internet y procrastinación en adolescentes y determinar si existe relación entre éstas.

Los resultados obtenidos destacan que los adolescentes se encuentran en una condición de riesgo ya que muestran un patrón de comportamiento temerario. Dicho patrón se caracteriza por la incesante búsqueda de experiencias excitantes y arriesgadas, adicción a las nuevas tecnologías digitales, en particular el internet, y el aplazamiento en la realización de actividades productivas y adaptativas.

Dichos hallazgos se confirman con los resultados obtenidos por diversas investigaciones (Clemente, Guzmán \& Salas, 2018; Rosabal et al., 2015; López, Toca, González, Pompa \& Alonso, 2020) destacando que los adolescentes constituyen un grupo de alto riesgo debido al deseo de experimentar vivencias novedosas e intensas; aunado a ello, muestran dificultad para controlar sus impulsos, condición que precede al comportamiento adictivo, ya que se ha referido que la impulsividad como una predisposición para actuar sin pensar en las consecuencias es la base de las adicciones incluyendo las psicológicas, como la dependencia hacia el internet. La dificultad en el autocontrol y la búsqueda de actividades placenteras que proporcionan recompensas casi inmediatas, son comunes en adolescentes procrastinadores, quienes aplazan tareas que conciben como aversivas e incluso aburridas.

Las conductas de riesgo estudiadas están relacionadas unas con otras, como lo demuestran los hallazgos, esto es, cuanto mayor es la búsqueda de experiencias novedosas y excitantes, mayor es 
la adicción al internet, lo que demuestra la necesidad de vivir los riesgos, incluso a través de las redes virtuales. El deseo de arriesgarse y de permanecer conectado a la tecnología, ocupan la atención y la mayor parte del tiempo del adolescente, lo que, a su vez, lleva al incumplimiento de objetivos vitales para su desarrollo, involucrándose cada vez más en conductas procrastinantes.

La asociación encontrada entre búsqueda de sensaciones y adicción al internet ha sido demostrada en diversas investigaciones (Rojas-Jara et al., 2018; Lara \& Pérez, 2014) donde se evidencia la existencia de factores de riesgo individuales que se vinculan a la adicción al internet, como la baja modulación de impulsos. Dicho rasgo está relacionado fuertemente con la búsqueda de experiencias intensas y excitantes, característica que aumenta la vulnerabilidad psicológica a las adicciones.

En la misma línea, las correlaciones halladas muestran que el rasgo búsqueda de sensaciones se asocia con la procrastinación. La literatura confirma tales hallazgos: cuando las personas procrastinan entran en conflicto entre lo que deberían hacer y lo que realmente quieren hacer, anticipando la tarea como poco atractiva y monótona, lo que las lleva a emprender acciones estimulantes y retardar el cumplimiento de objetivos; es por ello que se ha planteado que tareas que resultan aburridas y poco interesantes, promueven la procrastinación, siendo el talón de Aquiles de dicho rasgo la falla en el control de impulsos, que lleva a buscar experiencias placenteras e incumplir con tareas displancenteras (López et al., 2020; Gil, De besa \& Garzón, 2020; Steel, 2011).
En torno a la relación entre adicción al internet y procrastinación, los resultados obtenidos reflejaron que cuanto mayor sea el uso problemático de la tecnología, el adolescente aplaza más tareas. Efectivamente, dichos datos concuerdan con lo que arrojó el estudio de Díaz-Morales (2019), demostrando que el uso problemático del internet se asocia con la procrastinación, la cual expresa la falta de autorregulación de las personas que incurren en tal patrón, quienes se involucran con más frecuencia en tareas gratificantes a corto plazo, que se obtiene más rápidamente por medios virtuales.

En otro orden de ideas, se hallaron diferencias por sexo en búsqueda de sensaciones y adicción al internet. Los hombres puntuaron más alto en búsqueda de sensaciones, mientras que las mujeres muestran mayor dependencia hacia las redes virtuales. Dichos datos se alinean con los resultados obtenidos en diversas investigaciones, reportando que los hombres experimentan mayor necesidad de buscar sensaciones y experiencias excitantes lo cual podría deberse a factores biológicos y de socialización (Gil-Olarte, Cavalcante, Paramio, Zayas \& Gull, 2017; Latorre et al., 2014).

Con respecto a las diferencias en la adicción al internet por sexo, los datos coinciden con los publicados por Golpe, Gómez, Kim, Braña y Rial (2017), al destacar que las mujeres utilizan más la Red para buscar información sobre estudios y para el empleo de diversas aplicaciones sociales.

De igual manera, se encontraron diferencias por nivel educativo en búsqueda de sensaciones y procrastinación, siendo los adolescentes de secundaria 
quienes obtienen mayores puntajes en comparación con los adolescentes de preparatoria. En el caso particular de la procrastinación existe una controversia en los resultados a los que se ha llegado en diversas investigaciones acerca del papel que juega la edad en dicho patrón de comportamiento, ya que, por un lado, se reporta una disminución paulatina de la procrastinación con el aumento de la edad, pero, por otro, existe evidencia suficiente que sustenta que los alumnos tienden a procrastinar menos durante la educación secundaria, aumentando durante la preparatoria y el primer año de universidad (Díaz-Morales, 2019; Rodríguez \& Clariana, 2017).

Ambas aportaciones son válidas en el sentido de que es esperado que, conforme el adolescente madure, aumente el autocontrol; no obstante, cuando se comparan adolescentes de secundaria y preparatoria, dado que la etapa intermedia se caracteriza por una mayor intensidad de las emociones y mayor involucramiento en conductas de riesgo, lo común es que los alumnos de preparatoria muestren menor capacidad de planear y de regularse. Por lo anterior, conviene profundizar en las variantes de dicho comportamiento considerando no solo el grado escolar y la edad, sino también variables personales asociadas a rasgos de personalidad.

Finalmente, contrastando las diferencias encontradas en búsqueda de sensaciones por nivel educativo, los resultados concuerdan con estudios hechos en México que han reportado que la edad de inicio de conductas de riesgo es entre los 12 y 14 años de edad, observándose una percepción baja de riesgo de los adolescentes en esta etapa, a diferencia de los que se ubican entre los 15 y 17 años, siendo la necesidad de experimentar sensaciones novedosas e intensas el precedente de muchas de estas (Medina-Mora et al., 2015). Aun así, resulta indispensable ahondar en la diferenciación por etapas de la adolescencia sobre el constructo en cuestión.

Cabe destacar las limitaciones del estudio, observándose la necesidad de profundizar en las variables abordadas que permitan una caracterización más completa del fenómeno de estudio, considerando el impacto de factores de riesgo a nivel familiar y social. Así mismo, mejorar la obtención de la muestra de tal forma que sean incluidos mayor número de participantes por sectores de la ciudad de Morelia, a través de una muestra aleatoria que permita una mejor exploración, así como la inclusión de adolescentes de las etapas preadolescencia y tardía.

Los resultados obtenidos confirman la relación existente entre búsqueda de sensaciones, adicción al internet y procrastinación, concluyendo que los adolescentes muestran una tendencia a buscar experiencias excitantes e intensas, incluso a través de las nuevas tecnologías; el uso problemático del internet se traduce en una adicción donde la deficiente capacidad de autorregulación mantiene dicho patrón y se vincula con la falla en el cumplimiento de tareas, promoviendo conductas procrastinantes. Lo anterior se convierte en un círculo vicioso, ya que una conducta lleva a la otra, pero, a la vez, esta última actúa como predecesor de la primera. Hoy en día, los adolescentes son más vulnerables, ya que las conductas-problema esperadas dada la etapa evolutiva, tienen una alta probabilidad de transformarse en conductas de 
riesgo por la participación de diversos factores personales, familiares y sociales. Los datos obtenidos constituyen un acercamiento a un fenómeno complejo que requiere abordarse de manera multidisciplinaria para proponer estrategias que aminoren los riesgos, y promover el desarrollo de conductas más saludables en los adolescentes.

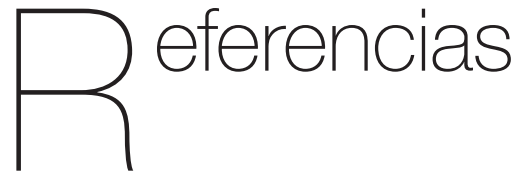

Arnett, J. (1994). Sensation seeking: A new conceptualization and a new scale. Personality and Individual Differences, 16(2), 289-296. Recuperado de https://www. researchgate.net/publication/222480310_ Sensation_seeking _ $A_{-} n e w_{-}$ conceptualization_and_a_new_scale.

Atayala, C. \& García, L. (2019). Procrastinación: Revisión teórica. Revista de Investigación en Psicología, 22(2), 363-378. http://dx.doi. org/10.15381/rinvp.v22i2.17435.

Barcelata, B. (2015). Adolescentes en riesgo. Una mirada desde la resiliencia. México: Manual Moderno.

Beranuy, M., Chamarro, A., Graner, C. \& Carbonell, X. (2009). Validación de dos escalas breves para evaluar la adicción a Internet y el abuso de móvil. Psicothema, 21(3), 480-485. Recuperado de https:// www.redalyc.org/pdf/727/72711821023. pdf.

Boyle, G. J., Saklofske, D. H., \& Matthews, G. (2015). Measures of personality and social psychological constructs. London, United Kingdom: Academic Press. Retrieved from https://www. researchgate.net/publication/291966548_ Measures_of_Personality_and_ Social_Psychological_Constructs/ link/56d25d4f08ae059e375fa11c/ download.

Busko, D. (1998). Causes and consequences of pefectionimsm and procrastination: A structural equation model. (Tesis de Maestría no publicada). University of Guelph, Ontario. Recuperado de http:// citeseerx.ist.psu.edu/viewdoc/download? doi=10.1.1.456.4450\&rep=rep1\&type=pdf.

Castaño, J.J. \& Páez, M.L. (2019). Familia y el uso y abuso de potenciales adictivos en jóvenes. Informes Psicológicos, 19(1), 105-118. http://dx.doi.org/10.18566/infpsic. v19n1a06.

Clemente, L., Guzmán, I. \& Salas, E. (2018). Adicción a redes sociales e impulsividad en universitarios de Cusco. Revista de Psicología Universidad Católica San Pablo, 8(1), 15-37. Recuperado de http://35.161.26.73/index.php/psicologia/ article/view/119/117.

Corona, F. \& Peralta, E. (2011). Prevención de conductas de riesgo. Revista Médica Clínica, 22(1), 68-75. Recuperado de https://www.sciencedirect.com/science/ article/pii/S0716864011703947.

Díaz-Morales, J. (2019). Procrastinación: una revisión de su medida y sus correlatos. Revista Iberoamericana de Diagnóstico y Evaluación RIDEP, 51(2), 43-60. Recuperado de https://www.aidep.org/ sites/default/files/2019-04/RIDEP51-Art4. pdf. 
Gámez, M. \&Villa, F. (2015). El modelo cognitivoconductual de la adicción a Internet: el papel de la depresión y la impulsividad en adolescentes mexicanos. Psicología y Salud, 25(1), 111-122. Recuperado de https://repositorio.uam.es/bitstream/ handle/10486/676537/modelo_gamez_ ps_2015.pdf?sequence=1\&isAllowed=y.

Gil, J., De Besa, M. \& Garzón, A. (2020). ¿Por qué procrastina el alumnado universitario? Análisis de motivos y caracterización del alumnado con diferentes tipos de motivaciones. Revista de Investigación Educativa, 38(1), 183-200. http://dx.doi. org/10.6018/rie.344781.

Gil-Olarte, P., Cavalcante, A., Paramio, A., Zayas, A. \& Gull, R. (2017). Impulsividad y búsqueda de sensaciones: implicaciones de inetrvencion en jóvenes. International Journal of Developmental and Educational Psychology. INFAD Revista de Psicología, 2(1), 393-404. https://doi.org/10.17060/ ijodaep.2017.n1.v2.952.

Golpe, S., Gómez, P., Kim, S., Braña, T. \& Rial, A. (2017). Diferencias de sexo en el uso de internet en adolescentes españoles. Psicología Conductual, 25(1), 129-146. Recuperado de https://www.researchgate. net/publication/317024119_Diferencias_ de_sexo_en_el_uso_de_internet_en_ adolescentes_espanoles.

González-Iglesias, B., Gómez-Fraguela, J., Gras, M. \& Planes, M. (2014). Búsqueda de sensaciones y consumo de alcohol: El papel mediador de la percepción de riesgos y beneficios. Anales de Psicología, 30(3), 1061-1068. http://dx.doi.org/10.6018/ analesps.30.3.170831.

Horvath, P. \& Zuckerman, M. (1996). Búsqueda de sensaciones, valoración y conducta de riesgo. Revista de Toxicomanías, 9, 26-39. Recuperado de https://www.catbarcelona.com/uploads/rets/RET09_3. pdf.

Lara, F. \& Pérez. G. (2014). Relación entre la variable búsqueda de sensaciones y la conducta de abuso de internet en adolescentes. Internacional Journal of Developmental and Educational Psychology, 26(1), 427-438. Recuperado de http://dehesa.unex.es/bitstream/ handle/10662/2055/0214-9877_2014_1_ 1_427.pdf?sequence $=4$ \&isAllowed $=\mathrm{y}$.

Latorre, P., Cámara, J. \& García, F. (2014). Búsqueda de sensaciones y hábitos de tabaquismo, consumo de alcohol y práctica deportiva en estudiantes de Educación Secundaria. Salud Mental, 37(2), 145-152. Recuperado de http://www.scielo.org.mx/ scielo.php?script=sci_arttext\&pid=S0185$33252014000200008 \& \operatorname{lng}=$ es\&tlng = es.

López, A., Toca, L., González, J., Pompa, B. \& Alonso, M. (2020). Reducción de la procrastinación académica mediante la Terapia de Aceptación y Compromiso: un estudio piloto. Clínica Contemporánea Revista de Diagnóstico Psicológico, Psicoterapia y Salud, 1(11), 1-16. https://doi. org/10.5093/cc2020a3.

Medina-Mora, M., Rafful, C., Villatoro, J., Oliva, N., Bustos, M. \& Moreno, M. (2015). Diferencias sociodemográficas entre usuarios de inhalables, usuarios de otras drogas y adolescentes no consumidores en una muestra mexicana de estudiantes. Revista Internacional de Investigación en Adicciones, 1(1), 6-15. https//:doi. org/10.28931/riiad.2015.1.02. 
Moreta-Herrera, R., Durán-Rodríguez, T. \& Villegas-Villacrés, N. (2018). Regulación Emocional y Rendimiento como predictores de la Procrastinación Académica en estudiantes universitarios. Revista de Psicología y Educación, 13(2), 155-166. https://doi.org/10.23923/rpye2018.01.166.

Oliva, A. (2007). Desarrollo cerebral y asunción de riesgos durante la adolescencia. Apuntes de Psicología, 25(3), 239-254. Recuperado de https://psicopedia.org/wpcontent/uploads/2014/06/Riesgos-en-laadolescencia.pdf.

Orcasita, L.T., Mosquera, J. A. \& Carrillo, T. (2018). Autoconcepto, autoeficacia y conductas sexuales de riesgo en adolescentes. Informes Psicológicos, 18(2), 141-168 http://dx.doi.org/10.18566/infpsic. v18n2a08.

Palacios, J. R. (2015). Propiedades psicométricas del inventario de búsqueda de sensaciones para adolescentes en México (IBS-Mx). International Journal of Psychological Research, 8(1), 46-60. Recuperado de http://www.scielo.org.co/ pdf/jpr/v8n1/v8n1a05.pdf.

Parra. V., Vargas, J., Zamorano, B., Peña, F., Velázquez, Y., Ruiz, L. \& Monreal, O. (2016). Adicción y factores determinantes en el uso problemático del internet, en una muestra de jóvenes universitarios EDUTEC. Revista Electrónica de Tecnología Educativa, 56, 60-73. https://doi.org/10.21556/ edutec.2016.56.741.

Pérez, J. \& Torrubia, R. (1986). Fiabilidad y validez de la versión española de la Escala de Búsqueda de Sensaciones (Forma V). Revista Latinoamericana de Psicología, 18(1), 7-22. Recuperado de https://www. redalyc.org/pdf/805/80518101.pdf
Ramos-Galarza, C., Jadán-Guerrero, J., Paredes-Núñez, L., Bolaños-Pasqueld, M. \& Gómez-García, A. (2017). Procrastinación, adicción al internet y rendimiento académico de estudiantes universitarios ecuatorianos. Estudios Pedagógicos, 43(3), 275-289. Recuperado de https:// scielo.conicyt.cl/scielo.php?pid=S071807052017000300016\&script=sci_arttext.

Rodríguez, A. \& Clariana, M. (2017). Procrastinación en Estudiantes Universitarios: su relación con la edad y el curso académico. Revista Colombiana de Psicología, 26(1), 45-60. https://doi. org/10.15446/rcp.v26n1.53572.

Rojas-Jara, C., Ramos-Vera, J., PardoGonzález, E. \& Henríquez-Caroca, F. (2018). Adicción a internet en adolescentes: una breve revisión. Drugs and Addictive Behavior, 3(2), 267-281. http://dx.doi. org/10.21501/24631779.2876

Rosabal, E., Romero, N., Gaquin, K. \& Hernández, R. (2015). Conductas de riesgo en los adolescentes. Revista Cubana de Medicina Militar, 44(2), 218-229. Recuperado de http://scielo.sld.cu/pdf/mil/ v44n2/mil10215.pdf.

Roth, M., Hammelstein, P. \& Brähler, E. (2007). Beyond a youthful behavior style. Age and sex differences in sensation seeking based on need theory. Personality and Individual Differences, 43(7), 1839-1850. https://doi. org/10.1016/j.paid.2007.06.004

Salas, E. (2014). Adicciones psicológicas y los nuevos problemas de salud. Cultura, 28, 111-146. Recuperado de https://www. researchgate.net/publication/272681780_ Adicciones_psicologicas_y_los_nuevos_ problemas_de_salud. 
Silva-Ortega, V. \& Zambrano-Villalba, C. (2018). Conducta adictiva al internet: redes sociales, uso del teléfono móvil y su incidencia en la personalidad. Revista Psicología UNEMI, 2(2), 41-49. Recuperado de http://181.188.214.100/index.php/ faso-unemi/article/view/700/622

Steel, P. (2011). Procrastinación. Por qué dejamos para mañana lo que podemos hacer hoy. Barcelona: Ed. Grijalbo.

Zuckerman, M., Eysenck, S. B., \& Eysenck, H. J. (1978). Sensation seeking in England and America: Cross-cultural, age, and sex comparisons. Journal of Consulting and Clinical Psychology, 46(1), 139-149. https:// doi.org/10.1037/0022-006X.46.1.139.

Zuckerman, M. (2007). Sensation seeking and risky behavior. Washington: American Psychological Association. https://psycnet. apa.org/doi/10.1037/11555-000. 